\title{
Review of: "Interferon Gamma Therapy in a Novel Case of Homozygous Interferon Alpha/beta Receptor Alpha Chain (IFNAR1) Deficiency Infected With SARS- CoV-2"
}

\section{Pankaj Chibber}

Potential competing interests: The author(s) declared that no potential competing interests exist.

The authors have unveiled a novel mutation and have presented a great therapy for combating COVID-19 in IFNARI negative patients. Although the work seems scientifically sound but I have some questions and comments that authors should address prior to publication.

1. As the study is based on a single observation, so question arises whether the results obtained are replicable or not. If yes how and why. Provide the data suggesting the replicability of the results.

2. As many other drugs were co-administered then how authors concluded that improvement in the clinical condition is due to IFN-Gamma. Authors should provide the control data where except IFN-Gamma other drugs were administered and no clinical improvement was observed in IFNAR1 negative patients. Add reference from literature if available. 